\title{
TWO SOLUBLE MODELS OF MAGNETIC SYSTEMS SHOWING CRITICAL POINTS OF HIGHER ORDER
}

\author{
Alex Hankey $^{*}$, T. S. Chang ${ }^{\dagger}$, and H. E. Stanley \\ Physics Department, Massachusetts Institute of Technology, \\ Cambridge, Massachusetts 02139
}

\section{ABSTRACT}

We give two soluble models which contain critical points of order four one order more complex than tricritical points or the tetracritical point presented to this conference two years ago by Nagle and Bonner. The models are one dimensional Ising models with a long-range interaction; one contains one staggered magnetic field, the other two such fields. The formal solutions are given.

The geometric theory of phase transitions and critical phenomena ${ }^{1}$ has led to the discovery of systems where several lines of critical points intersect. 2 Points where three lines of critical points meet have been called tricritical points ${ }^{2}$, and points where four lines of critical points meet, tetracritical points ${ }^{3}$ and so on ${ }^{4}$.

It has been shown ${ }^{5}$, however, that in the particular case considered by Nagle and Bonner, which was reported on at this conference 6 two years ago, the tetracritical point is a point on a single smooth curve of tricritical points from which it is topologically indistinguishable. This is because tricritical points are the endpoints of lines of points where three phases are in equilibrium and the same is true of the tetracritical point. A more complex point will be the endpoint of a line of points where four (or more) phases are in equilibrium, and which is also the endpoint of lines of tricritical points. A necessary condition for the existence of a more complex point is, therefore, a line of points where four phases coexist and this is not satisfied by the Nagle-Bonner $\operatorname{model}^{5}$.

A new classification of critical points using strictly topological quantities has therefore been proposed 5,7 . Critical points are classified by an index called the order $\mathscr{O}$, which turns out to be equal to the dimensionality $\mathbf{n}$ of the total space ${ }^{8}$ of field variables, minus the dimensionality $d$ of the critical space considered: $\mathscr{O}=\mathrm{n}$-d. Thus for ordinary critical points $\mathscr{O}=2$, and for tricritical points (and the Nagle-Bonner tetracritical point) $\mathscr{O}=3.9$ To find a critical point of order 4 , it is necessary to find a system where four phases coexist on a line of points for varying temperature; furthermore, the end of this line of points must also be the endpoint of four lines of tricritical points. This critical point will be topologically different from and one dimension less than the lines of tricritical points.

* Supported by a Lindemann Fellowship. Present address: Stanford Linear Accelerator Center.

$\dagger$ Also at North Carolina State University. 
Motivated by these considerations we have found several systems containing lines of tricritical points. Among them are two exactly soluble one dimensional Ising models 10 which contain intersecting lines of tricritical points and thus 7 a critical point of order four. The solutions of these models are presented here.

The first model is a variation on the model of Nagle and Bonner. To make four phases stable in the region $\mathrm{T}>0$ (two ferromagnetic and now two antiferromagnetic instead of a single disordered phase), we split the long-range interaction so that it acts separately on the two sublattices of odd numbered and even numbered spins. The Hamiltonian is:

$$
\begin{gathered}
\mathscr{H}=-\mathrm{J}_{\mathrm{SR}} \sum_{\mathrm{i}} \mathrm{s}_{\mathrm{i}} \mathrm{s}_{\mathrm{i}+1}-\sum_{\mathrm{i}, \mathrm{r}} \mathrm{J}(\mathrm{r}) \mathrm{s}_{2 \mathrm{i}} \mathrm{s}_{2 \mathrm{i} \pm 2 \mathrm{r}^{-}} \sum_{\mathrm{i}, \mathrm{r}} \mathrm{J}(\mathrm{r}) \mathrm{s}_{2 \mathrm{i}+1} \mathrm{~s}_{2 \mathrm{i} \pm 2 \mathrm{r}+1} \\
-\mathrm{H} \sum_{\mathrm{i}} \mathrm{s}_{\mathrm{i}}-\mathrm{H}_{2} \sum_{\mathbf{i}}(-)^{\mathrm{i}+1} \mathrm{~s}_{\mathbf{i}}
\end{gathered}
$$

Here $\mathrm{H}_{2}$ is the staggered magnetic field of wavelength 2 lattice sites and $J(r)$ is the usual long-range interaction $J(r)=\operatorname{Lim}(\gamma \rightarrow 0)$ a $\gamma \mathrm{e}^{-\gamma r}$. This Hamiltonian has an important discrete symmetry which will necessarily be reflected by the phase diagram of the solution of the model. It is defined by the operation $\mathrm{s}_{\mathrm{i}} \rightarrow(-)^{\mathbf{i}} \mathrm{s}_{\mathrm{i}}, \mathrm{H} \rightarrow \mathrm{H}_{2}, \mathrm{H}_{2} \rightarrow \mathrm{H}, \mathrm{J}_{\mathrm{SR}} \rightarrow-\mathrm{J}_{\mathrm{SR}}$. That means that the solution for positive $\mathrm{J}_{\mathrm{SR}}$ is related to that for negative $\mathrm{J}_{\mathrm{SR}}$ by

$$
\mathrm{G}\left(\mathrm{H}, \mathrm{H}_{2}, \mathrm{~T} ;{ }_{\mathrm{SR}}\right)=\mathrm{G}\left(\mathrm{H}_{2}, \mathrm{H}, \mathrm{T} ;-\mathrm{J}_{\mathbf{S R}}\right)
$$

so that $\mathrm{M}\left(\mathrm{H}, \mathrm{H}_{2}, \mathrm{~T} ;+\mathrm{J}_{\mathrm{SR}}\right)=\mathrm{M}_{2}\left(\mathrm{H}_{2}, \mathrm{H}, \mathrm{T} ;-\mathrm{J}_{\mathrm{SR}}\right)$. In particular the well$\mathrm{known}^{3}$ lines of tricritical points in the $\mathrm{J}_{\mathrm{SR}}, \mathrm{H}, \mathrm{T}$ hyperplane for $\mathrm{J}_{\mathrm{SR}}<0$ will be complimented by lines of tricritical points in the $\mathrm{J}_{\mathrm{SR}}, \mathrm{H}_{2}$, $\mathrm{T}$ hyperplane for $\mathrm{J}_{\mathrm{SR}}>0$ : these will intersect on the $\mathrm{T}$ axis which therefore passes through a critical point of order four. The partition function for the Hamiltonian (1) is easily calculated using the same methods as Nagle and Bonner $^{3}$. The additional complication of a second long-range interaction is easily dealt with by defining magnetisations. $\mathrm{M}^{\mathrm{O}}=\sqrt{2} \sum \mathrm{s}_{2 \mathrm{i}+1}, \mathrm{M}^{\mathrm{E}}=$ $\sqrt{2} \sum \mathrm{s}_{2 \mathrm{i}}$ and the corresponding fields $\mathrm{H}^{\mathrm{O}}=\left(\mathrm{H}+\mathrm{H}_{2}\right) / \sqrt{2}, \mathrm{H}^{\mathrm{E}}=\left(\mathrm{H}-\mathrm{H}_{2}\right) / \sqrt{2}$. The Gibbs function can now be thought of either as a function of $\mathrm{H}, \mathrm{H}_{2}$ or of $\mathrm{H}^{\mathrm{O}}, \mathrm{H}^{\mathrm{E}}$. The Gibbs function is first calculated with zero long-range interaction. This gives $\mathrm{G}_{\mathrm{NN}}\left(\mathrm{H}_{0}, \mathrm{H}_{20}, \mathrm{~T}\right)$ (the same as in reference 3 ); the corresponding Helmholtz potential is then given by $A_{N N}\left(M, M_{2}, T\right)=$ $\mathrm{G}_{\mathrm{NN}}\left(\mathrm{H}_{0}, \mathrm{H}_{20}, \mathrm{~T}\right)+\mathrm{MH}_{0}+\mathrm{M}_{2} \mathrm{H}_{20}$. Note that by our definitions $\mathrm{MH}+\mathrm{M}_{2} \mathrm{H}_{2}=$ $\mathrm{MOHO}+\mathrm{MEH}_{\mathrm{H}} \mathrm{E}$. The effects of the long-range interaction can now be subtracted off as $\dot{E}_{\mathrm{L} R}=-\mathrm{a}\left(\mathrm{MO}^{2}+\mathrm{M}^{\mathrm{E} 2}\right)=-\mathrm{a}\left(\mathrm{M}^{2}+\mathrm{M}_{2}^{2}\right)$ Hence the solution of the problem is given by

$$
A\left(M, M_{2}, T\right)=C E\left\{G_{N N}\left(H_{0}, H_{20}, T\right)+M_{0}+M_{2} H_{20}-a\left(M^{2}+M_{2}^{2}\right)\right\}
$$

where $\mathrm{CE}$ denotes convex envelope. The physical fields $\mathrm{H}, \mathrm{H}_{2}$ minimise $\mathrm{A}-\mathrm{MH}-\mathrm{M}_{2} \mathrm{H}_{2}$ giving 


$$
\begin{aligned}
\mathrm{H} & =\mathrm{H}_{0}-2 \mathrm{aM} \\
\mathrm{H}_{2} & =\mathrm{H}_{20}-2 \mathrm{aM}_{2}
\end{aligned}
$$

At the special point 4 lines of tricritical points, bounding 6 surfaces of critical points intersect. For $\mathrm{JSR}_{\mathrm{SR}}=0$ the interaction is the same as in the Weiss model - and the same exponents result. This is in contradiction with the analysis of multicritical point exponents provided by Theumann and Hoye 11 .

The second model follows a suggestion of reference 4 and introduces a second staggered magnetic field - one of wavelength four lattice sites $\mathrm{H}_{4}$. This simplifies the calculation because we only have to treat a block of four spins. ${ }^{12}$ The Hamiltonian is now

$$
\begin{aligned}
\mathscr{H}= & -J_{S R} \sum_{i} s_{i} s_{i+1}-\sum_{s_{i, r}} J(r) s_{i} s_{i \pm r} \\
& -H \sum_{i} s_{i}-H_{2} \sum_{i}(-)^{i+1} s_{i}-H_{4} \sum_{i} c_{i} s_{i}
\end{aligned}
$$

where $c_{i}=+1$ if $i=(4 n+1),(4 n+2) ; c_{i}=-1$ if $i=(4 n+3), 4 n$. This is solved by the usual methods applied to one dimensional Ising models. 3,4 First it is solved without the long-range interaction by introducing four $2 \times 2$ transfer matrices representing interactions between spins $(4 n+1,4 n+2) \ldots$ $(4 n, 4 n+1)$.

$$
\begin{aligned}
\mathbf{T}^{1} & =\left[\begin{array}{l}
\operatorname{Exp}\left[\beta\left(\mathrm{J}+\mathrm{H}+\mathrm{H}_{4}\right)\right] \operatorname{Exp}\left[\beta\left(-\mathrm{J}+\mathrm{H}_{2}\right)\right] \\
\operatorname{Exp}\left[\beta\left(-\mathrm{J}-\mathrm{H}_{2}\right)\right] \operatorname{Exp}\left[\beta\left(\mathrm{J}-\mathrm{H}-\mathrm{H}_{4}\right)\right.
\end{array}\right]=\left[\begin{array}{l}
\operatorname{Exp}[\beta(\mathrm{J}+\mathrm{H})] \operatorname{Exp}\left[\beta\left(-\mathrm{J}-\mathrm{H}_{2}+\mathrm{H}_{4}\right)\right] \\
\operatorname{Exp}\left[\beta\left(-\mathrm{J}+\mathrm{H}_{2}-\mathrm{H}_{4}\right)\right] \operatorname{Exp}[\beta(\mathrm{J}-\mathrm{H})]
\end{array}\right] \\
\mathbf{T}^{3} & =\left[\begin{array}{l}
\operatorname{Exp}\left[\beta\left(\mathrm{J}+\mathrm{H}-\mathrm{H}_{4}\right)\right] \operatorname{Exp}\left[\beta\left(-\mathrm{J}+\mathrm{H}_{2}\right)\right] \\
\operatorname{Exp}\left[\beta\left(-\mathrm{J}-\mathrm{H}_{2}\right)\right] \operatorname{Exp}\left[\beta\left(\mathrm{J}-\mathrm{H}+\mathrm{H}_{4}\right)\right]
\end{array}\right] \mathrm{T}^{4}=\left[\begin{array}{l}
\operatorname{Exp}[\beta(\mathrm{J}+\mathrm{H})] \operatorname{Exp}\left[\beta\left(-\mathrm{J}-\mathrm{H}_{2}-\mathrm{H}_{4}\right)\right] \\
\operatorname{Exp}\left[\beta\left(-\mathrm{J}+\mathrm{H}_{2}+\mathrm{H}_{4}\right)\right] \operatorname{Exp}[\beta(\mathrm{J}-\mathrm{H})]
\end{array}\right]
\end{aligned}
$$

These are multiplied together to give the $2 \times 2$ matrix $M$ acting between neighboring blocks of four spins. The larger eigenvalue $\lambda^{+}$of this matrix will give the partition function when the number of blocks of spins becomes infinite. The Gibbs function per spin is then given by

$$
\mathrm{G}\left(\mathrm{H}, \mathrm{H}_{2}, \mathrm{H}_{4}, \mathrm{~T}\right)=\frac{\mathrm{kT}}{4} \ln \lambda^{+}=\frac{1}{4} \mathrm{kT} \ln (\mathrm{X}+\sqrt{\mathrm{X}+\mathrm{Det}})
$$

where Det $=\operatorname{Det} M=\left(e^{2 \beta J}-e^{-2 \beta J}\right)^{4}$.

$$
\begin{aligned}
\mathrm{X}=\mathrm{e}^{4 \beta \mathrm{J}} \mathrm{Ch}(4 \beta \mathrm{H}) & +\mathrm{e}^{-4 \beta \mathrm{J}} \mathrm{Ch}\left(4 \beta \mathrm{H}_{2}\right)+1+\mathrm{Ch}\left(4 \beta \mathrm{H}_{4}\right)+ \\
& +4 \mathrm{Ch}(2 \beta \mathrm{H}) \operatorname{Ch}\left(2 \beta \mathrm{H}_{2}\right) \operatorname{Ch}\left(2 \beta \mathrm{H}_{4}\right)
\end{aligned}
$$


The same method as for the first model is now applied, but as for Nagle and Bonner, one must take the convex envelope not of the full Helmholtz potential $A\left(M, M_{2}, M_{4}, T\right)$ but of the mixed function $G_{1}\left(M, H_{2}, H_{4}, T\right)$ so that

$$
\mathrm{G}_{1}\left(\mathrm{M}, \mathrm{H}_{2}, \mathrm{H}_{4}, \mathrm{~T}\right)=\left\{\mathrm{CE} \mathrm{G}\left(\mathrm{H}_{0}, \mathrm{H}_{20}, \mathrm{H}_{40}, \mathrm{~T}\right)+\mathrm{H}_{0} \mathrm{M}-\mathrm{aM}^{2}\right\}
$$

where the three magnetic fields are related to the bare fields by

$$
\mathrm{H}=\mathrm{H}_{0}-2 \mathrm{aM}, \quad \mathrm{H}_{2}=\mathrm{H}_{20}, \mathrm{H}_{4}=\mathrm{H}_{40}
$$

An analysis of the $T=0$ plane of this model ${ }^{5}$ reveals points where 5 and 6 phases coexist. The former is the end of 3 lines where 3 phases coexist and one line where 4 coexist. As $T$ is increased these lines sweep out surfaces of points which must be bounded for increasing $T$. The boundaries should be lines of critical points of order 3 and they will intersect at a critical point of order 4.

\section{REFERENCES}

1. R. B. Griffiths and J. C. Wheeler, Phys. Rev. A , 1047 (1970).

2. R. B. Griffiths, Phys. Rev. Letters $24,715(19 \overline{70})$.

3. J. F. Nagle, Phys. Rev. A2, 2124 (1) $\overline{9} 70)$.

J. F. Nagle and J. C. Bonner, J. Chem. Phys. 54, 729 (1971).

4. W. K. Theumann and J. S. Hoye, J. Chem. Phys. 55, 4159 (1971).

5. A. M. A. Hankey, Ph. D. Thesis, MIT (1972).

6. J. C. Bonner and J. F. Nagle, J. App. Phys. 42, 1280 (1971).

7. T. S. Chang, A. Hankey and H. E. Stanley (a preprint).

8. The word space means here, a smooth connected subspace of the total space of thermodynamic field variables.

9. Generally $\mathscr{O}$ is equal to the number of independent vector directions out of a critical space and may in principle be equaled to the number of directions of scaling.

10. The models were proposed by considering the $T=0$ phase diagrams, a method that is reported in detail in reference 5.

11. Specifically, Theumann and Hoye suggested on the last page of their paper (reference 4) that where s critical points converge $\beta=1 / 2 \mathrm{~s}$. But in the case given here $s=6$ and $\beta=1 / 2$.

12. The number of lattice sites that must be considered is the LCM of all the wavelengths of the staggered fields introduced. The smallest LCM of two positive integers greater than one is $4=\operatorname{LCM}(2,4)$. (LCM means lowest common multiple.) 